\title{
Comments on conformal masses, asymptotic backgrounds and conservation laws
}

\author{
Nathalie Deruelle* \\ Institut des Hautes Etudes Scientifiques, \\ 35 Route de Chartres, 91440 , Bures-sur-Yvette, France \\ Joseph Katz ${ }^{\dagger}$ \\ The Racah Institute of Physics, Edmond Safra Campus \\ Givat Ram, Jerusalem 91904, Israel
}

August 12, 2018

\begin{abstract}
The "conformal mass prescriptions" were used recently to calculate the mass of spacetimes in higher dimensional and higher curvature theories of gravity. These definitions are closely related to Komar integrals for spacetimes that are conformally flat at great distances from the sources. We derive these relations without using the conformal infinity formalism.
\end{abstract}

\section{(i) Conformal mass and Komar integral}

In a recent paper Gibbons, Perry and Pope 21] used the prescription given in Ashtekar and Magnon [7] and in Ashtekar and Das 4] to calculate the mass of a rotating black hole in "anti-de Sitter backgrounds in higher dimensions [because] all the ambiguities that plague the Komar prescription are avoided". Okuyama and Koga 32] similarly generalized the AshtekarMagnon-Das prescription to higher curvature gravitational fields for different reasons, that is : "the conformal completion technique provides an intrinsic and background independent definition of conserved quantities in an asymptotically AdS spacetime".

Now, Ashtekar and Magnon-Ashtekar [6] for asymptotically flat spacetimes and Magnon 30] for asymptotically anti-de Sitter spacetimes showed how to derive the "conformal" mass and angular momentum from the Komar integral [29. The derivation was made entirely in the conformal infinity formalism. Let us do it here in the following way.

*email:deruelle@ihes.fr

†email: jkatz@phys.huji.ac.il 
The Komar superpotential ${ }^{1} \hat{J}_{K}^{\mu \nu}$ is an anti-symmetric tensor density defined as:

$$
\hat{J}_{K}^{\mu \nu} \equiv \frac{1}{\kappa} D^{[\mu} \hat{\xi}^{\nu]} \quad \text { with } \quad \kappa \equiv \frac{8 \pi G}{c^{4}},
$$

where $\xi^{\mu}$ is any vector field. Let $d S_{\mu \nu}=\frac{1}{2} \epsilon_{\mu \nu \rho \sigma} d x^{\rho} \wedge d x^{\sigma}$ be the coordinate surface element of a closed surface $S$. The Komar integral is

$$
K \equiv \frac{1}{2} \oint_{S} \hat{J}_{K}^{\mu \nu} d S_{\mu \nu}
$$

The gist of the relation between Komar's integral and the Ashtekar-Magnon prescription, which involves the Weyl tensor, is easily understood as follows. Let $r^{2} d S$ (with $d S=$ $\sin \theta d \theta d \varphi$ ) be the surface element of a sphere of radius $r$ in the asymptotically flat background in spherical coordinates. ${ }^{2}$ Then $K$ is a finite quantity if its integrant is of the form

$$
K=\oint_{r \rightarrow \infty} f(r) r^{2} d S=\oint_{r \rightarrow \infty}\left(\frac{a}{r^{2}}+\frac{b}{r^{3}}+\cdots\right) r^{2} d S
$$

where $a, b, \cdots$ are functions of $\theta$ and $\varphi$. Now $K$ may equally well be written as

$$
K=\oint_{r \rightarrow \infty}\left(-\frac{1}{2} r \frac{\partial f}{\partial r}\right) r^{2} d S
$$

The covariant form of the integrant in (44) must thus be proportional to $-\frac{1}{2} m^{\rho} D_{\rho} \hat{J}_{K}^{\mu \nu}$, where $m^{\rho}$ is the unit vector in the radial direction normal to the sphere. It can only be linear in the curvature tensor components that is, away from the sources where the Ricci tensor vanishes, in the components of the Weyl tensor.

Let us show that in an explicitly covariant way.

\section{(ii) Asymptotically flat spacetime}

If $\xi^{\mu}$ is a Killing vector field, the covariant derivative of Komar's superpotential is related to the Riemannian curvature tensor $R_{\rho \sigma}^{\mu \nu}$ as follows :

$$
D_{\rho} \hat{J}_{K}^{\mu \nu}=\frac{1}{\kappa} \hat{g}^{\sigma[\mu} D_{\rho \sigma} \xi^{\nu]}=-\frac{1}{\kappa} \hat{R}_{\rho \sigma}^{\mu \nu} \xi^{\sigma}
$$

Let $n^{\mu}$ and $m^{\nu}$ be two orthogonal normalized vectors orthogonal to $S$ :

$$
g^{\mu \nu} n_{\mu} n_{\nu}=1 \quad, \quad g^{\mu \nu} m_{\mu} m_{\nu}=-1 \quad \text { and } \quad g^{\mu \nu} m_{\mu} n_{\nu}=0 .
$$

\footnotetext{
${ }^{1}$ Indices $\lambda, \mu, \nu, \rho, \cdots=0,1,2,3$; the metric $g_{\mu \nu}\left(x^{\lambda}\right)$ has signature +--- and $g$ is its determinant. Multiplication by $\sqrt{-g}$ is indicated by an additional hat, $\hat{X}$, on a previously defined symbol like $X$. $D$ is the covariant derivative associated with the metric $g_{\mu \nu}$. The Levi-Civita symbol is $\epsilon_{\mu \nu \rho \sigma}$ with $\epsilon_{0123}=1$. Square brackets denote antisymmetrisation: $X_{[\mu \nu]} \equiv \frac{1}{2}\left(X_{\mu \nu}-X_{\nu \mu}\right)$.

${ }^{2}$ We shall see below how to extend this argument to an asymptotically anti-de Sitter spacetime.
} 
The coordinate surface element $d S_{\mu \nu}$ may be written as $d S_{\mu \nu}=-2 n_{[\mu} m_{\nu]} d^{2} x$ where $d^{2} x$ is thus defined as $d^{2} x=n^{\mu} m^{\nu} d S_{\mu \nu}$. We therefore have, for any Killing vector field $\xi^{\mu}$ :

$$
\frac{1}{2} m^{\rho}\left(D_{\rho} \hat{J}_{K}^{\mu \nu}\right) d S_{\mu \nu}=\frac{1}{\kappa} \hat{R}_{\mu \nu \rho \lambda} \xi^{\lambda} n^{\mu} m^{\nu} m^{\rho} d^{2} x .
$$

$\xi^{\mu}$ may only be an asymptotic Killing vector of time translations in which case (5) and (7) are only asymptotically true. In asymptotically static and spherical coordinates $(t, r, \theta, \varphi)$ in which $\xi^{\lambda} \rightarrow(1,0,0,0), n^{\nu} \rightarrow(1,0,0,0), m^{\nu} \rightarrow(0,1,0,0), \sqrt{-g} \rightarrow r^{2} \sin \theta, d^{2} x=d \theta d \varphi$, $d S=\sin \theta d \theta d \varphi$ and $g_{00} \rightarrow 1-2 G M /\left(c^{2} r\right)$ (see e.g. [31]), a short calculation yields :

$$
\lim _{r \rightarrow \infty}\left[-\frac{1}{2} r \oint_{r \rightarrow \infty} \frac{1}{2} m^{\rho}\left(D_{\rho} \hat{J}_{K}^{\mu \nu}\right) d S_{\mu \nu}\right]=\frac{1}{2} \oint \hat{J}_{K}^{\mu \nu} d S_{\mu \nu}=\frac{1}{2} M c^{2},
$$

which, following (3) and (4), is the expected result connecting the Komar integral and its derivative. Eq. (8) shows as is well known that Komar's integral yields half the expected value of the mass. ${ }^{3}$

The connection between the integral of the radial derivative of Komar's superpotential and the Ashtekar-Hansen [5] conformal mass is also very simply obtained. Since $\xi^{\mu} \rightarrow n^{\mu}$, we have, from eq. (8) with (7):

$$
M c^{2}=\lim _{r \rightarrow \infty}\left[-r \oint_{r \rightarrow \infty} \frac{1}{\kappa}\left(m^{\rho} m^{\sigma} R_{\mu \rho \sigma \nu}\right) n^{\mu} n^{\nu} r^{2} d S\right] .
$$

Outside the sources, the Ricci tensor vanishes by virtue of Einstein's equations and $R_{\nu \rho \sigma}^{\lambda}=$ $C_{\nu \rho \sigma}^{\lambda}$, where $C_{\nu \rho \sigma}^{\lambda}$ is the Weyl tensor. At conformal flat infinity the Ashtekar-Hansen conformal factor $\Omega \rightarrow 1 / r^{2}$. We may therefore rewrite (9) as follows:

$$
M c^{2}=-\frac{1}{2} \frac{c^{4}}{G} \oint_{r \rightarrow \infty} E_{\mu \nu}\left(\Omega^{-1} n^{\mu} n^{\nu}\right) \frac{d S}{4 \pi} \quad \text { in which } \quad E_{\mu \nu}=\Omega^{\frac{1}{2}} m^{\rho}\left(\Omega^{-1} m^{\sigma}\right) C_{\mu \rho \sigma \nu} .
$$

This expression is, with slightly different notations (in particular our metric is denoted without a hat), that given in Ashtekar and Magnon [6] on page 796 where they relate the conformal mass to the Komar integral in exactly the same way but in a conformal infinity formulation.

\section{(iii) Asymptotically anti-de Sitter spacetime}

When spacetime is asymptotically anti-de Sitter, the Komar integral (2) diverges. A way to remedy this is "renormalization": one subtracts from $\hat{J}_{K}^{\mu \nu}$ the Komar superpotential of the anti-de Sitter background $\overline{\hat{J}_{K}^{\mu \nu}}$ (see [30] or [27]):

$$
\hat{J}_{K}^{\mu \nu}-\overline{\hat{J}_{K}^{\mu \nu}} \equiv \frac{1}{\kappa} D^{[\mu} \hat{\xi}^{\nu]}-\frac{1}{\kappa} \overline{D^{[\mu} \hat{\xi}^{\nu]}},
$$

\footnotetext{
${ }^{3}$ One may claim that this is a matter of normalization. See, however, section (iv).
} 
where barred quantities are defined in the anti-de Sitter background spacetime with metric $\bar{g}_{\mu \nu}\left(x^{\lambda}\right){ }^{4}$

If $\xi^{\mu}$ and $\bar{\xi}^{\mu}$ are Killing vector fields we have, as above:

$$
\frac{1}{2}\left(m^{\rho} D_{\rho} \hat{J}_{K}^{\mu \nu}-\overline{m^{\rho} D_{\rho} \hat{J}_{K}^{\mu \nu}}\right) d S_{\mu \nu}=\frac{1}{\kappa}\left(\hat{R}_{\mu \nu \rho \lambda} \xi^{\lambda} n^{\mu} m^{\nu} m^{\rho}-\overline{\hat{R}_{\mu \nu \rho \lambda} \xi^{\lambda} n^{\mu} m^{\nu} m^{\rho}}\right) d^{2} x .
$$

The metrics $g_{\mu \nu}$ and $\bar{g}_{\mu \nu}$ coincide at infinity and $\bar{R}_{\mu \nu \rho \sigma}=-\frac{1}{l^{2}}\left(\bar{g}_{\rho \nu} \bar{g}_{\mu \sigma}-\bar{g}_{\sigma \nu} \bar{g}_{\mu \rho}\right)$ where $l^{2}$ is the characteristic scale of the anti-de Sitter spacetime. In static and spherically symmetric coordinates $(t, r, \theta, \varphi)$ :

$$
d \bar{s}^{2}=\bar{g}_{\mu \nu} d x^{\mu} d x^{\nu}=\left(1+r^{2} / l^{2}\right) d t^{2}-\frac{d r^{2}}{1+r^{2} / l^{2}}-r^{2}\left(d \theta^{2}+\sin ^{2} \theta d \varphi^{2}\right) .
$$

If $\xi^{\mu} \rightarrow \bar{\xi}^{\mu}$ is the asymptotic Killing vector of time translations we have :

$$
\xi^{\lambda} \rightarrow \bar{\xi}^{\lambda}=(1,0,0,0) \quad, \quad n^{\nu} \rightarrow \bar{n}^{\nu} \simeq \frac{l}{r}(1,0,0,0) \quad \text { and } \quad m^{\nu} \rightarrow \bar{m}^{\nu} \simeq \frac{r}{l}(0,1,0,0) .
$$

As for the asymptotic behavior of the needed metric components $g_{\mu \nu}$, we have [30]:

$$
g_{00} \rightarrow \bar{g}_{00}-\frac{2 G M}{c^{2} r} \quad, \quad g_{11} \rightarrow \bar{g}_{11}-\frac{2 G M l^{4}}{c^{2} r^{5}}
$$

$S$ being again the sphere of radius $r \rightarrow \infty$. One thus gets, see [16]:

$$
\frac{1}{2} \oint_{r \rightarrow \infty}\left(\hat{J}_{K}^{\mu \nu}-\overline{\hat{J}_{K}^{\mu \nu}}\right) d S_{\mu \nu}=\frac{1}{2} M c^{2}
$$

Notice that the non-zero component of $m^{\rho}$ is not 1 but $r / l$. Thus $m^{\rho} D_{\rho}$ already contains the necessary factor $r$ which appears in (4) but has also an unnecessary factor $l$ which we remove by multiplication and we obtain therefore :

$$
-\frac{1}{2} l \oint_{r \rightarrow \infty} \frac{1}{2}\left(m^{\rho} D_{\rho} \hat{J}_{K}^{\mu \nu}-\overline{m^{\rho} D_{\rho} \hat{J}_{K}^{\mu \nu}}\right) d S_{\mu \nu}=\frac{1}{2} M c^{2}
$$

This is the expected result connecting the renormalized Komar integral (16) and its derivatives (17).

The connection between (17) and the Ashtekar-Magnon-Das conformal mass formula is again very simply obtained. We have

$$
M c^{2}=-l \oint_{r \rightarrow \infty} \frac{1}{\kappa} m^{\rho} m^{\sigma}\left(R_{\mu \rho \sigma \nu}-\bar{R}_{\mu \rho \sigma \nu}\right) n^{\mu} \xi^{\nu} r^{2} d S .
$$

\footnotetext{
${ }^{4}$ For a detailed definition of what is meant by a background and the mapping on physical spacetime, see e.g. 9. Let us emphasize that the background is defined in a coordinate independent way. For instance, for a Kerr spacetime on an anti-de Sitter background the background metric is $\bar{g}_{\mu \nu}=g_{\mu \nu}(m=a=0)$ in whatever coordinates $g_{\mu \nu}$ has been written.
} 
Outside the sources the Einstein equations are $R_{\mu \nu}=3 g_{\mu \nu} / l^{2}$ and imply $R_{\mu \rho \sigma \nu}=C_{\mu \rho \sigma \nu}-$ $\frac{1}{l^{2}}\left(g_{\mu \nu} g_{\rho \sigma}-g_{\mu \sigma} g_{\nu \rho}\right)$. Hence, at infinity, $\left(R_{\mu \rho \sigma \nu}-\bar{R}_{\mu \rho \sigma \nu}\right) \rightarrow C_{\mu \rho \sigma \nu}$ because there, $g_{\mu \nu}=\bar{g}_{\mu \nu}$ and $\bar{C}_{\mu \rho \sigma \nu}=0$. Thus (18) can also be written as:

$$
M c^{2}=-\frac{l}{8 \pi} \frac{c^{4}}{G} \oint_{r \rightarrow \infty}\left(m^{\rho} m^{\sigma} C_{\mu \rho \sigma \nu}\right) \xi^{\mu} n^{\nu} r^{2} d S .
$$

This formula is the Ashtekar-Magnon-Das 4] 30. 4] formula which reads, in the (slightly adapted) notations of 32 :

$$
M c^{2}=-\frac{l}{8 \pi} \frac{c^{4}}{G} \oint \hat{\mathcal{E}}_{\mu \nu} \xi^{\mu} \hat{N}^{\nu} \sqrt{\hat{\sigma}} d^{2} x \quad \text { with } \quad \hat{\mathcal{E}}_{\mu \nu}=l^{2} \lim _{\Omega \rightarrow \infty}\left(\Omega \hat{M}^{\rho} \hat{M}^{\sigma} C_{\mu \rho \sigma \nu}\right) .
$$

Here $\sqrt{\hat{\sigma}} d^{2} x \equiv d S, \hat{N}^{\nu}=l \xi^{\nu} \equiv r n^{\nu}$, with $n^{\nu}$ defined in (14); the conformal factor $\Omega \rightarrow 1 / r$, $\hat{M}^{\rho} \equiv(r / l) m^{\rho}$, with $m^{\rho}$ defined in (14), so that $\hat{\mathcal{E}}_{\mu \nu} \equiv r\left(m^{\rho} m^{\sigma} C_{\mu \rho \sigma \nu}\right)$.

\section{(iv) Comments}

The Ashtekar-Hansen and Ashtekar-Magnon-Das global conservation laws are based on the Penrose formalism [33] in which asymptotic regions are described by a conformal spacetime with an appropriate conformal factor $\Omega$ which depends on the underlying asymptotic physical metric.

We saw that those prescriptions amount to computing the radial derivative of Komar's integral $K$. For isolated stationary sources in asymptotically flat spacetimes if $\xi^{\mu}$ is the Killing vector of time translations $K=\frac{1}{2} M c^{2}$ but if $\xi^{\mu}$ is a Killing vector of spatial rotations then $K=c J$ where $J$ is the corresponding component of the angular momentum. This factor 2 discrepancy is the Komar anomalous factor [33]. (Ashtekar and Hansen formula is normalized to yield the right mass.) In an asymptotically anti-de Sitter spacetime $K$ diverges. This is the Komar integral "ambiguity" alluded to in [21]. As we recalled, this divergence is easily removed if instead of $K$ we use [27] $K-\bar{K}$ where $\bar{K}$ is the Komar integral evaluated in the anti-de Sitter "background". The anomalous factor 2 however remains. Finally, at null infinity $(u \equiv t-r=$ const.) $K$ does not [41] give the Penrose 34] mass nor is $d K / d u$ the Bondi [11 mass loss. Thus, the Komar integral does not describe properly conserved quantities associated with asymptotic symmetries ${ }^{5}$. Notice also that the Ashtekar-MagnonDas conformal prescription depends on second derivatives of the metric and requires thus more information on the background than the Komar integral.

\footnotetext{
${ }^{5}$ Conservation laws are associated with global spacetime symmetries and a variational principle. The Komar superpotential is a construct with an arbitrary field and is not related to a variational principle (see however below) or to global symmetries. The same is true for the Ashtekar et al. constructs.
} 
In spite of its limitations the Komar superpotential played an important role in the development of conservation laws in general relativity and in the discovery of the first law of black hole thermodynamics [8]. It is also fair to say that the conformal mass and conservation laws of Ashtekar et al. appeared before the limitations of Komar integrals became apparent and were corrected.

The Komar superpotential [26] can be derived from the Hilbert Lagrangian (the scalar curvature) using traditional Noether identities. It is a characteristic property of global conservation laws in general relativity derived from any Lagrangian by means of these identities [23] that they depend on a vector field $\xi^{\mu}$ and that conserved vector densities, say $\hat{J}^{\mu}$, are divergences of anti-symmetric tensor densities or superpotentials : $\hat{J}^{\mu}=\partial_{\nu} \hat{J}^{\mu \nu}$, like the Komar superpotential $\hat{J}_{K}^{\mu \nu}$. The asymptotic behavior of the gravitational field is also an essential ingredient in the construction of globally conserved quantities. In most cases spacetimes become flat at great distances from the sources but there has also been great interest in asymptotically anti-de Sitter spacetimes. In any cases it is useful to regard spacetimes at great distances from the sources as backgrounds with a metric of their own. In global considerations we are not interested in how spacetimes are mapped on backgrounds at finite distances. Backgrounds are just another convenient and covariant way to deal with asymptotic limits.

There are, however, well known difficulties with superpotentials in that they are not uniquely defined. Recent work by Silva [38, Julia and Silva [24] and Chen and Nester [13] 14] based on ideas of Regge and Teitelboim 36] lifted such ambiguities by imposing reasonable conditions that make $\hat{J}^{\mu \nu}$ essentially unique. That unique KBL superpotential ${ }^{6}$ [26], 27] is:

$$
\hat{J}^{\mu \nu} \equiv \hat{J}_{K}^{\mu \nu}-\bar{J}_{K}^{\mu \nu}+\frac{1}{\kappa} \xi^{[\mu} \hat{k}^{\nu]} \quad \text { with } \quad \hat{k}^{\nu} \equiv \frac{1}{\sqrt{-g}} \bar{D}_{\mu}\left(-g g^{\mu \nu}\right) .
$$

According to Julia and Silva 24] this superpotential is the only one possessing all the following properties. It is generally covariant and can be computed in any coordinate system. In cartesian coordinates of an asymptotically flat spacetime it gives the ADM mass formula [2] and in asymptotically anti-de Sitter spacetimes it gives the AD mass [1]. It gives the mass and angular momentum as well as the Brown and Henneaux conformal charges [10] with the right normalization in any dimensions $D \geq 3$. It can also be used for any Killing vector field

\footnotetext{
${ }^{6}$ It has also been shown [25] that the criterion used to define the KBL superpotential is indeed equivalent to the covariant simplectic phase space methods of Wald [40, Iyer and Wald [22, Witten [42] and Ashtekar, Bombelli and Reula [3].
} 
of the background. It reproduces the Penrose mass [33] the Bondi mass loss [12], the Penrose linear momentum [33, the Sachs linear momentum flux 37] and the Penrose 34] and Dray and Streubel 20] angular momentum at null infinity. One may add that it gives the mass of a Kerr black hole in an anti-de Sitter background in $D$ dimensions [15]. Finally the extension of the KBL prescription to Einstein-Gauss-Bonnet theory of gravity [16] gives also the mass and angular momenta of rotating black holes on AdS backgrounds in that theory [17.

Of course, if one is interested in calculating conservation laws at spatial infinity only, either flat or AdS, one can as well use the ADM superpotential [2] or the Abbot and Deser one [1] or the Petrov and Katz one [35] or, in Einstein-Gauss-Bonnet theory, the Deser and Tekin one [18], 19] or other covariant Hamiltonian formulations many of which are reviewed in the recent paper of Szabados [39].

\section{Acknowledgements}

N.D. thanks the Hebrew University for hospitality and financial support.

Note added in proof : In this paper we limited ourselves to a comparison between the Komar and Ashtekar et al. masses. It is important to note that equation (9) (or (10)) where $n^{\mu}$ is replaced by $\xi_{\phi}^{\nu}=(0,0,0,1)$ gives also the right value of the Kerr angular momentum: $J=$ Mac. Similarly equation (19) (or (20)) gives the right Kerr AdS mass and angular momentum, as shown explicitely in [28]. Hence defining the mass and angular momentum by means of the derivative of the Komar superpotential (or, equivalently, as we have shown, by means of equations (10) and (20)) cures the problem of the anomalous factor 2 which plagues the Komar integrals. (Note however that Ashtekar Hansen and Das [4] [5] use a different construct for the angular momentum.) We are grateful to Jun-ichirou Koga for bringing our attention to that point.

\section{References}

[1] Abbott L F and Deser S 1982 Stability of gravity with a cosmological constant Nucl. Phys. B 195, 76

[2] Arnowitt R, Deser S and Misner C W 1960 Energy and the criteria for radiation in general relativity Phys. Rev. $\mathbf{1 1 8} 1100$ 
[3] Ashtekar A, Bombelli L and Reula O 1990 The covariant phase space of asymptotically flat gravitational fields in Analysis, geometry and mechanics: 200 years after Lagrange Ed. Francaviglia M and Holm D (Amsterdam: North Holland) p. 417

[4] Ashtekar A and Das S 2000 Asymptotic anti-de Sitter spacetimes: conserved quantities Class. Quantum Grav. 17 L17

[5] Ashtekar A and Hansen R O 1978 A unified treatment of null and spatial infinity in general relativity. I. Universal structure, asymptotic symmetries, and conserved quantities at spatial infinity J. Math. Phys. 191542

[6] Ashtekar A and Magnon Ashtekar A 1979 On conserved quantities in general relativity J. Math. Phys. 20793

[7] Ashtekar A and Magnon A 1984 Asymptotically anti-de Sitter spacetimes Class. Quantum Grav. 1 L39

[8] Bardeen J M, Carter B and Hawking S W 1973 The four laws of black hole mechanics Commun. Math. Phys. 31161

[9] Bičák J and Katz J 2005 On the uniqueness of harmonic coordinates Czech. J. Phys. 55105 (Preprint gr-qc/0503018)

[10] Brown J D and Henneaux M 1986 Central Charges in the canonical realization of asymptotic symmetries : An example from three-dimensional gravity Commun. Math. Phys. 104207

[11] Bondi H 1960 Gravitational waves in general relativity Nature 186535

[12] Bondi H, van der Burg M G J and Metzner A K W 1962 Gravitational waves in general relativity VII Waves from axi-symmetric isolated systems Proc. Roy. Soc. London A 26921

[13] Chen C M and Nester J M 1999 Quasilocal quantities for GR and other gravity theories Class. Quantum Grav. 161279 (Preprint gr-qc/9809020)

[14] Chen C M and Nester J M 2000 A simplectic Hamiltonian derivation of quasi-local energy-momentum for GR Grav. Cosmol. 6257 (Preprint gr-qc/0001088) 
[15] Deruelle N and Katz J 2005 On the mass of a Kerr-anti-de Sitter spacetime in $D$ dimensions Class. Quantum Grav. 22421 (Preprint gr-qc/0410135)

[16] Deruelle N, Katz J and Ogushi S 2004 Conserved charges in Einstein Gauss-Bonnet theory Class. Quantum Grav. 211971 (Preprint gr-qc/0310098)

[17] Deruelle N and Morisawa Y 2005 Mass and angular momenta of Kerr anti-de Sitter spacetimes in Einstein-Gauss-Bonnet theory Class. Quantum Grav. 22933 (Preprint gr-qc/0410135)

[18] Deser S and Tekin B 2002 Gravitational Energy in Quadratic Curvature Gravities Phys. Rev. Lett. 89101101 (Preprint hep-th/0205318)

[19] Deser S, Kanik I and Tekin B 2005 Conserved Charges of Higher D Kerr-AdS Spacetimes Class. Quantum Grav. 22 L103 (Preprint gr-qc/0506057)

[20] Dray T and Streubel M 1984 Angular momentum at null infinity Class. Quantum Grav. 115

[21] Gibbons G W, Perry M J and Pope C N 2005 The first law of thermodynamics for Kerranti-de Sitter black holes Class. Quantum Grav. 221503 (Preprint hep-th/0408217)

[22] Iyer V and Wald R M 1994 Some properties of Nœther charge and a proposal for dynamical black hole entropy Phys. Rev. D 50846 (Reprint gr-qc/9403028)

[23] Julia B and Silva S 1998 Currents and superpotentials in classical gauge theories: I. Local results with applications to perfect fluids and general relativity Class. Quantum Grav. 152173 (Preprint gr-qc/9804029)

[24] Julia B and Silva S 2000 Currents and superpotentials in classical gauge theories: II. Global aspects and the example of affine gravity Class. Quantum Grav. 174733 (Preprint gr-qc/005127)

[25] Julia B and Silva S 2002 On covariant phase space methods (Preprint hep-th/0205072)

[26] Katz J 1985 A note on Komar's anomalous factor Class. Quantum Grav. 2423

[27] Katz J, Bičák J and Lynden-Bell D 1997 Relativistic conservation laws and integral constraints for large cosmological perturbations Phys. Rev. D 555957 (Reprint gr-qc/0504041) 
[28] Koga J., The first law of AdS black holes in higher curvature gravity, (Reprint hep-th/05005219)

[29] Komar A 1959 Covariant conservation laws in general relativity Phys. Rev. 113934

[30] Magnon A 1985 On Komar integrals in asymptotically anti-de Sitter spacetimes J. Math. Phys. 263112

[31] Misner C W, Thorne K S and Wheeler J A 1973 Gravitation (San Francisco: Freeman) p. 604

[32] Okuyama N and Koga J 2005 Asymptotically anti-de Sitter spacetimes and conserved quantities in higher curvature gravitational theories Phys. Rev. D $\mathbf{7 1} 084009$ (Preprint hep-th/0501044)

[33] Penrose R 1964 Conformal treatment of infinity in Relativity Groups and Topology Ed. DeWitt C and DeWitt B (London: Blackie and son Ltd) p. 565

[34] Penrose R 1982 Quasi-local mass and angular momentum in general relativity Proc. Roy. Soc. London A 38153

[35] Petrov A N and Katz J 2002 Conserved currents, superpotentials and cosmological perturbations Proc. Roy. Soc. London A 458319 (Preprint gr-qc/9911025)

[36] Regge T and Teitelboim C 1974 Role of surface integrals in the formulation of General Relativity Annals of Physics $\mathbf{8 8} 286$

[37] Sachs R K 1962 Gravitational Waves in General Relativity. VIII. Waves in Asymptotically Flat Space-Time Proc. Roy. Soc. London A $\mathbf{2 7 0} 103$

[38] Silva S. 1999 On superpotentials and charge algebras of gauge theories Nucl. Phys. B 558391 (Preprint hep-th/9809109)

[39] Szabados L B 2004 Quasi-local energy-momentum and angular momentum in GR: A review article Living Reviews in Relativity 7 2004-4, revised edition 2005

[40] Wald R M 1993 Black Hole Entropy is Nœther Charge Phys. Rev. D 483427 (Reprint gr-qc/9307038) 
[41] Winicour J H and Tamburino L 1965 Lorentz-covariant gravitational energymomentum linkages Phys. Rev. Lett. 15601

[42] Witten E 1986 Interacting field theory of open superstrings Nucl. Phys. B 276291 